\title{
Facile Exfoliation of 3D Pillared Metal-Organic Frameworks (MOFs) to Produce MOF Nanosheets with Functionalized Surfaces
}

Ji-Hua Deng, $,{ }^{\dagger,}, \#$ Ya-Qiong Wen ${ }^{\dagger, \# ~ J e r e m y ~ W i l l m a n, ~}{ }^{\S}$ Wen-Ju Liu, ${ }^{\ddagger}$ Yun-Nan Gong, ${ }^{\dagger}$ Di-Chang Zhong, ${ }^{\dagger}+* *$ Tong-Bu Lu, ${ }^{\ddagger *}$ and Hong-Cai Zhou ${ }^{* *}$

${ }^{\dagger}$ College of Chemistry \& Chemical Engineering, Gannan Normal University, Ganzhou, 341000, P. R. China. E-mail: zhong_dichang@hotmail.com Institute for New Energy Materials and Low Carbon Technologies, Joint Laboratory of MOE International Cooperation in Materials Microstructure, School of Materials Science and Engineering, School of Chemistry and Chemical Engineering, Tianjin University of Technology, Tianjin 300384, China. E-mail: lutongbu@tjut.edu.cn

$\S$ Texas A\&M University, Department of Chemistry, College Station, TX 77842 USA.

E-mail: zhou@chem.tamu.edu

\#These authors contributed equally to this work. 


\section{Experimental Section}

Concentration determination of MOF nanosheets. $5 \mathrm{~mL}$ of the suspension containing MOF nanosheets was allowed to naturally evaporate. The resulting solid was weighed, and the concentration of MOF nanosheets was calculated by the mass of the solid dividing volume $(5 \mathrm{~mL})$. For MOF nanosheet 1, $16.0 \mathrm{mg}$ of nanosheets was obtained, so the concentration of nanosheet $\mathbf{1}$ is $3.2 \mathrm{mg} / \mathrm{mL}$, and for MOF nanosheet $\mathbf{2}, 13.0 \mathrm{mg}$ of nanosheets was obtained, so the concentration of nanosheet $\mathbf{2}$ is 2.6 $\mathrm{mg} / \mathrm{mL}$.

Powder X-ray diffraction measurements. After stirred for five days, the resulting solution was allowed to stand overnight. Then $15 \mathrm{~mL}$ of the upper clear solution was transferred to a culture dish and naturally dried at room temperature. The afforded solid (merging solid obtained from several control experiments) was placed on a flat glass plate used for XRD measurement, with the $2 \theta$ of $5-35^{\circ}$ and the step length of $0.2 \%$ step.

SEM measurements. After stirred for a certain period, $0.5 \mathrm{~mL}$ of the upper clear solution was naturally dried at room temperature. Then a small amount of nanosheets were mounted rigidly to a copper holder with a conductive adhesive. The sample was sprayed with gold to enhance its conductivity, and scanned under vacuum mode with an acceleration voltage of $20 \mathrm{kV}$.

AFM measurements. $0.5 \mathrm{~mL}$ of the upper clear solution was spread on a mica substrate, naturally dried at room temperature, and scanned on a Bruker FastScan Bio atomic force microscope (AFM).

Water contact angle measurements. The upper clear solution was naturally dried at room temperature. The resulting powder was collected and further dried in a vacuum oven at $50{ }^{\circ} \mathrm{C}$ for 12 hours, to make sure the sample was dried enough. The powder 
sample was spread on a flat glass substrate and smoothed by pressing with another flat glass plate. The water contact angle measurements of the prepared samples were carried out on a TX500H rotating drop interface tension meter. 
<smiles>c1cc(-c2ccncc2)ccn1</smiles><smiles>Cc1ccncc1</smiles><smiles>Cc1cccc(C)n1</smiles><smiles>Cc1cc(C)nc(C)c1</smiles>

Figure S1. a, The bpy pillared ligand within $\mathbf{1}$ and $\mathbf{2}$, and $\mathbf{b}-\mathbf{d}$, the replacing ligands used to exfoliate pillared 3D MOFs.
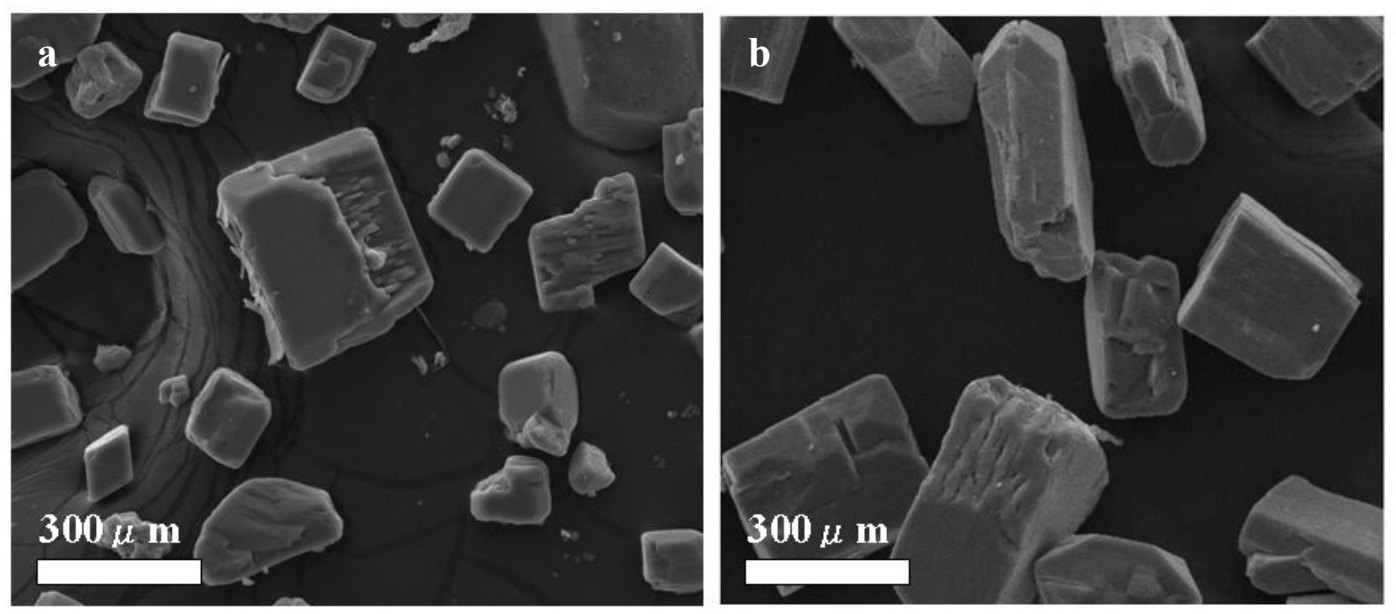

Figure S2. SEM images of MOFs a, $\mathbf{1}$ and $\mathbf{b}, \mathbf{2}$. 
$\mathbf{a}$

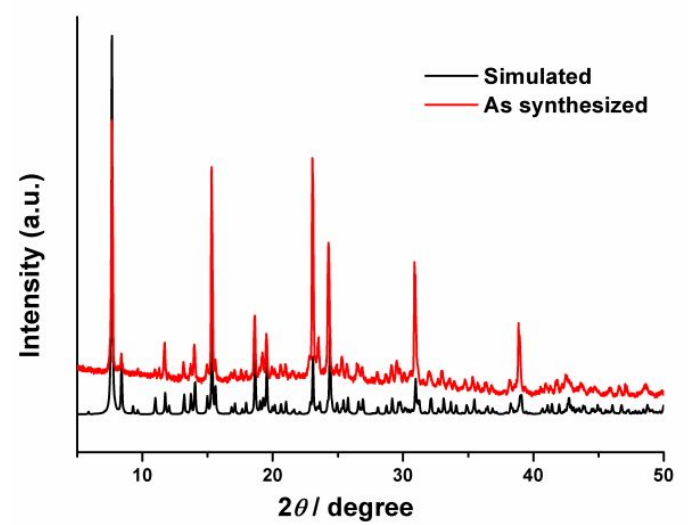

b

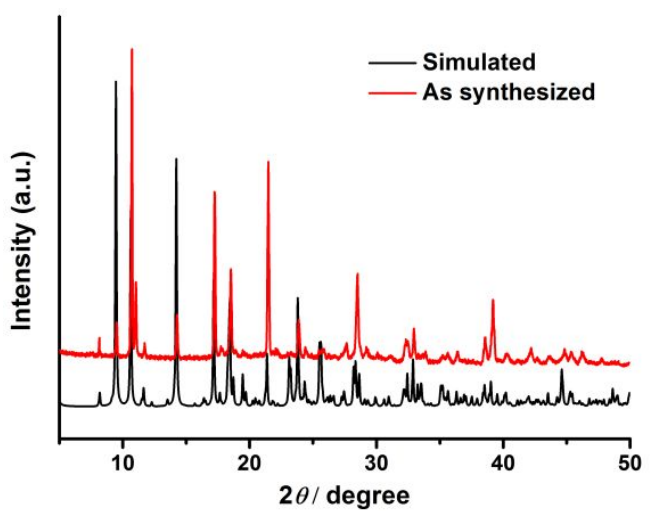

Figure S3. Powder XRD patterns of synthesized MOFs of $\mathbf{a}, \mathbf{1}$ and $\mathbf{b}, \mathbf{2}$.
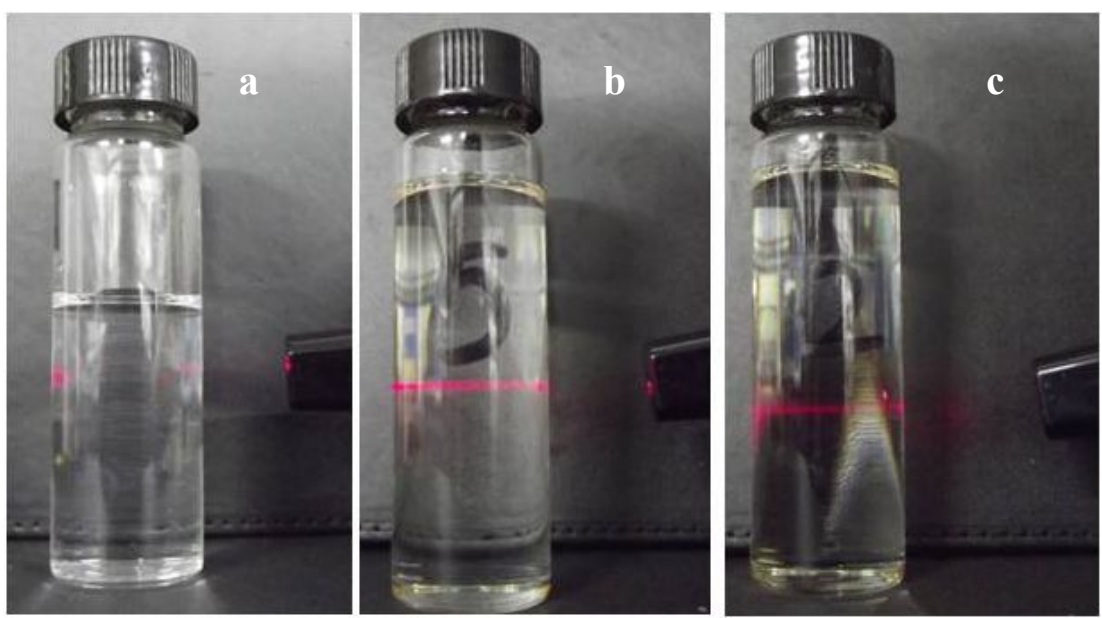

Figure S4. The path of the red laser beam through a, 4-methylpyridine solution, and 4-methylpyridine solution containing b, 1 and $\mathbf{c}, 2$. 
a

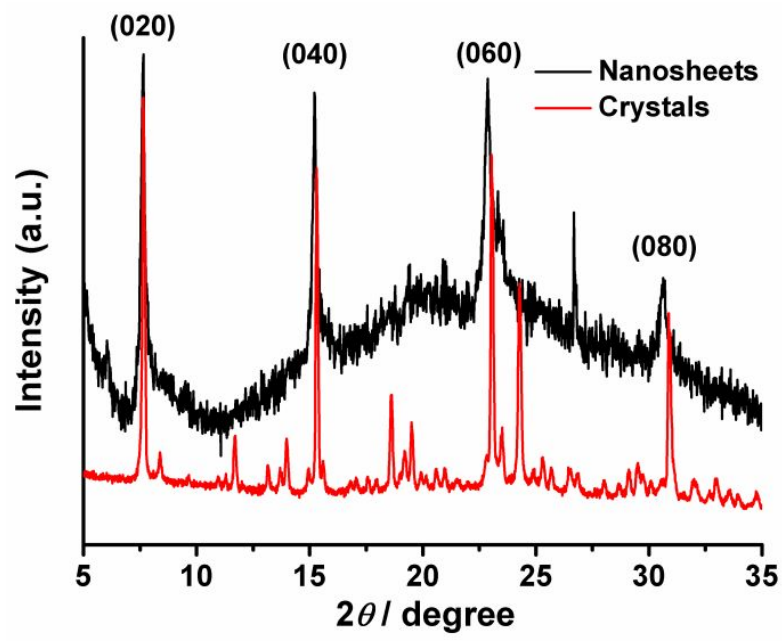

b

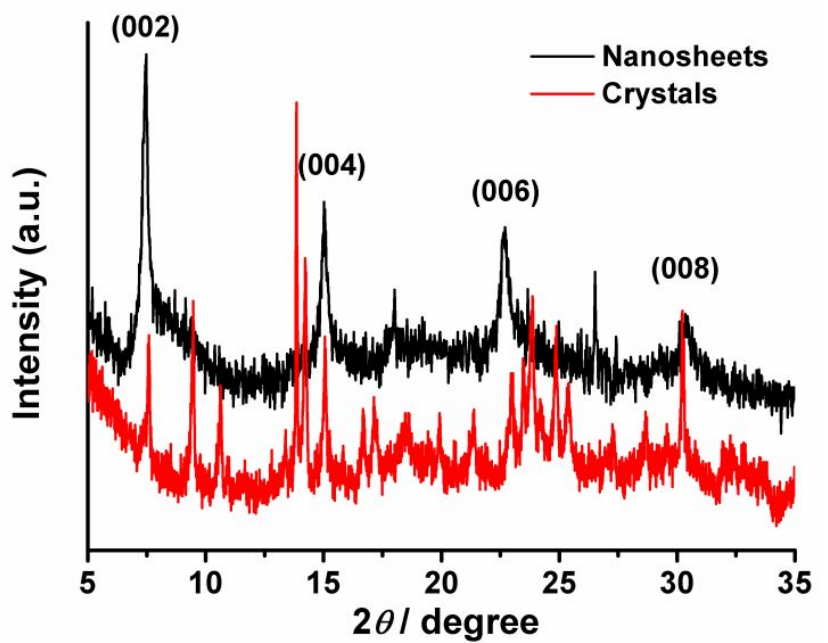

Figure S5. XRD patterns of the crystals and nanosheets for $\mathbf{a}, \mathbf{1}$ and $\mathbf{b}, \mathbf{2}$. 
a

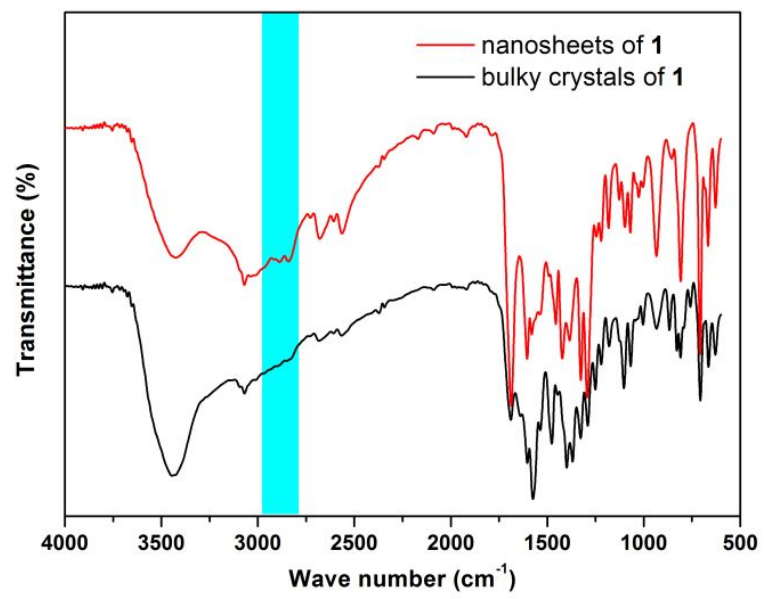

b

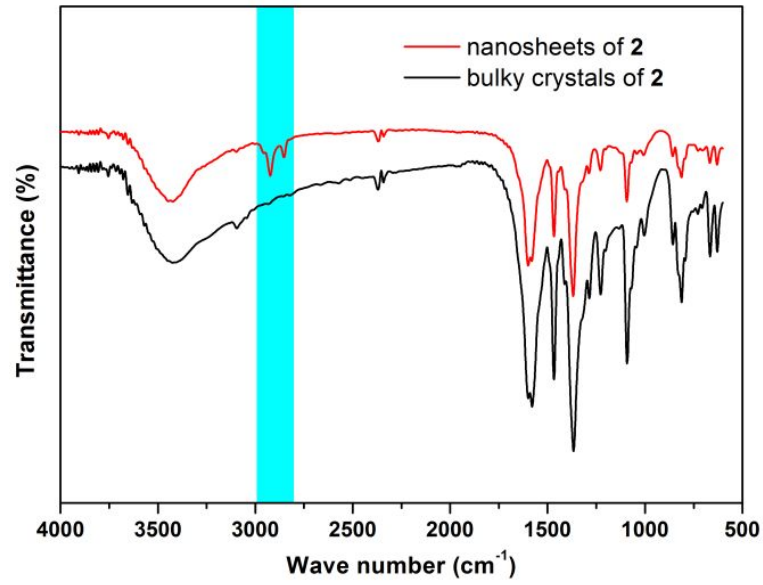

Figure S6. IR spectra of bulky crystals and MOF nanosheets of $\mathbf{a}, \mathbf{1}$ and $\mathbf{b , 2}$. 
a

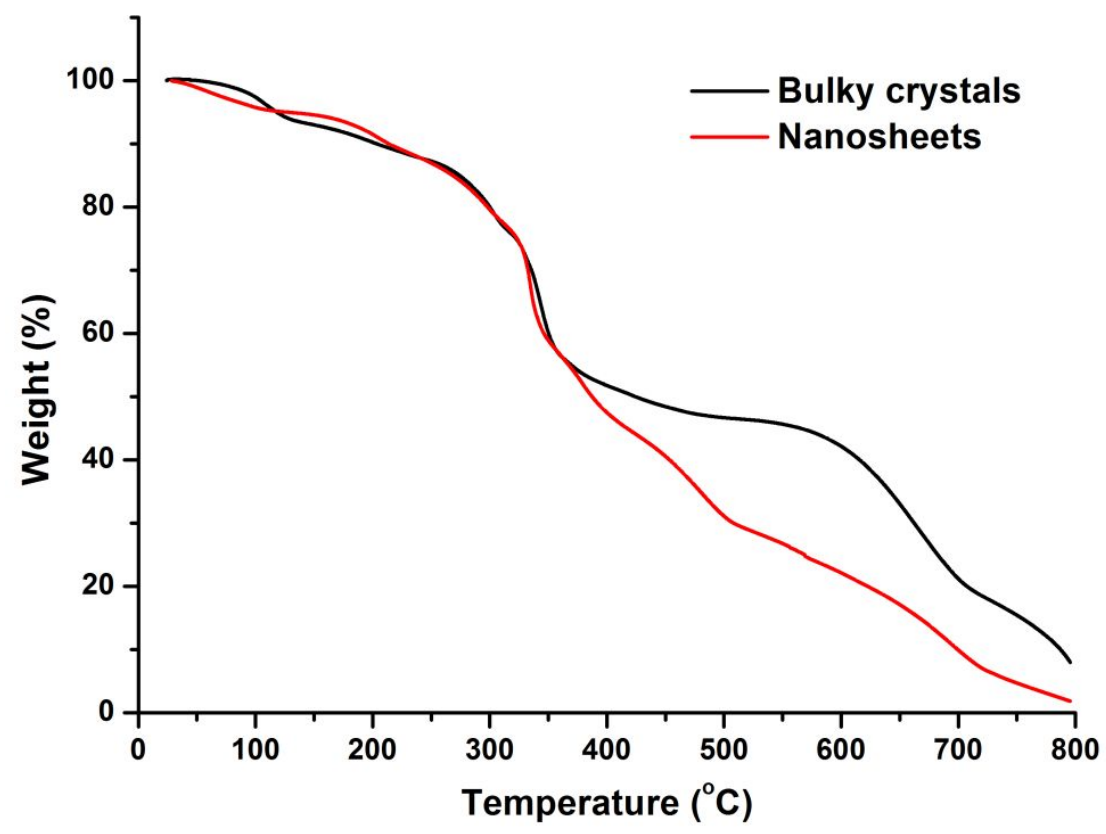

b

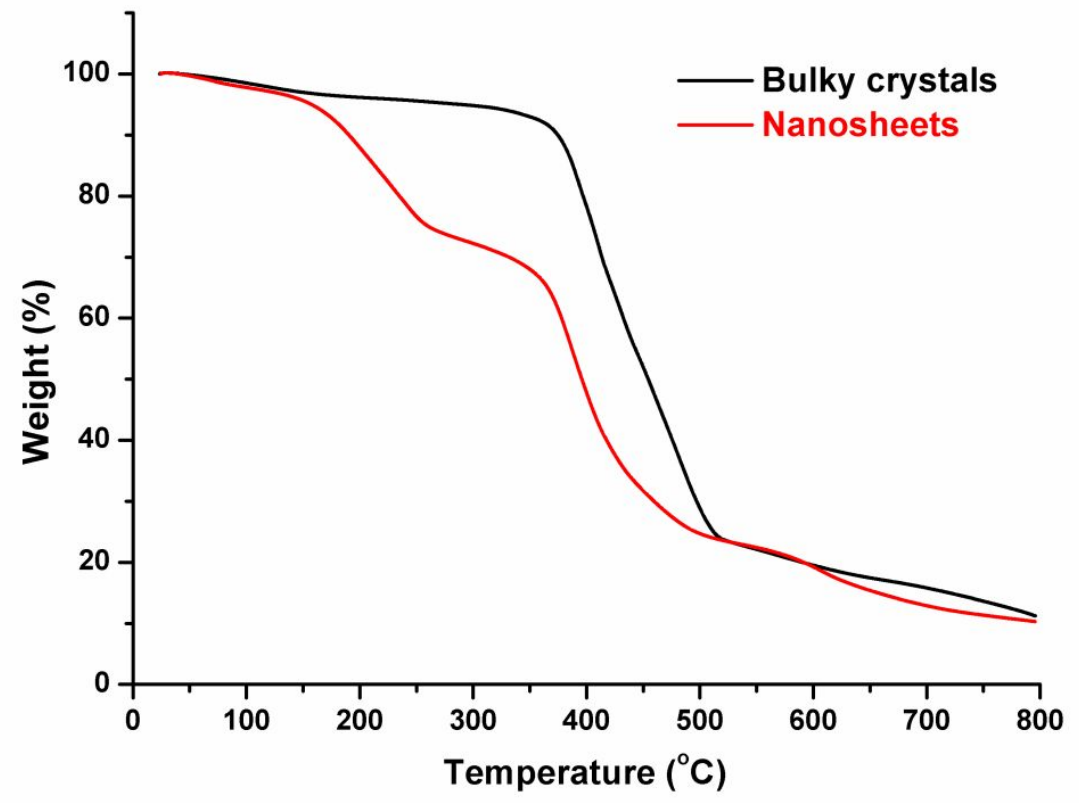

Figure S7. TG curves of bulky crystals and MOF nanosheets of $\mathbf{a}, \mathbf{1}$ and $\mathbf{b}, \mathbf{2}$. 

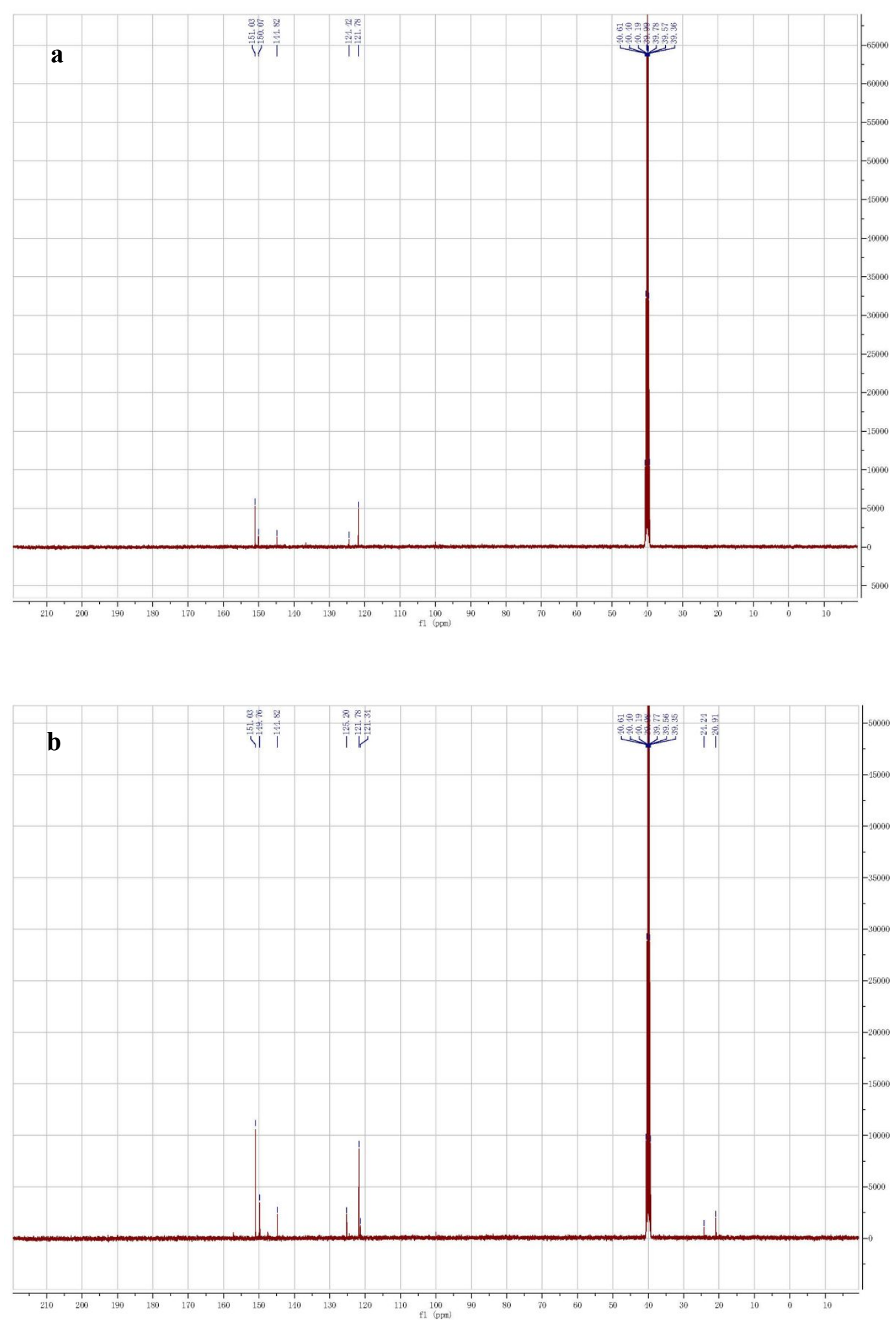

Figure S8. ${ }^{13} \mathrm{C}$ NMR of $\mathbf{a}$, the bulky crystals of $\mathbf{1}$ and $\mathbf{b}$, the exfoliated nanosheets of 1 in DMSO- $d_{6}(400 \mathrm{MHz}, 298 \mathrm{~K})$ after sonicated for $24 \mathrm{~h}$. 
a
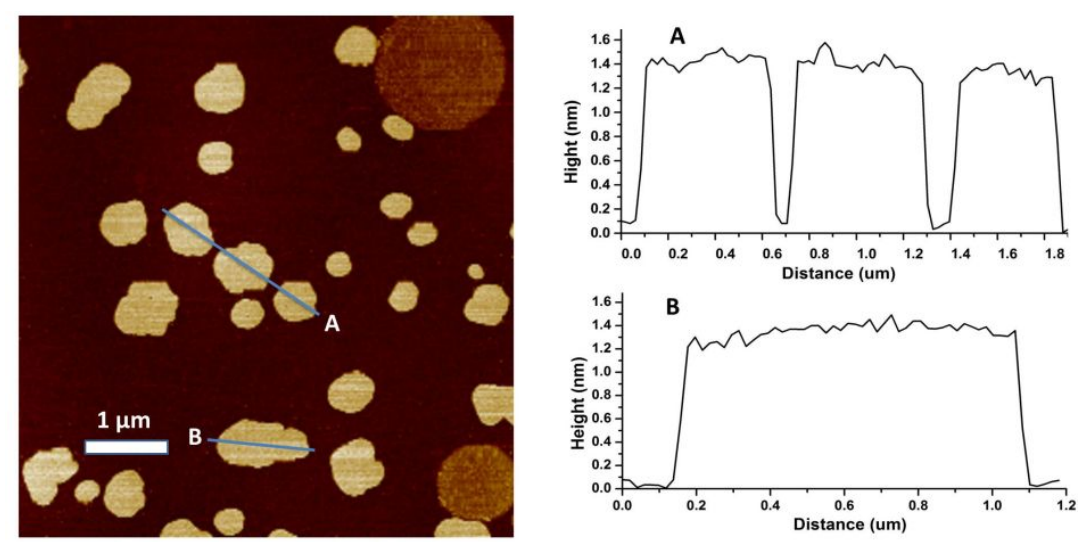

b
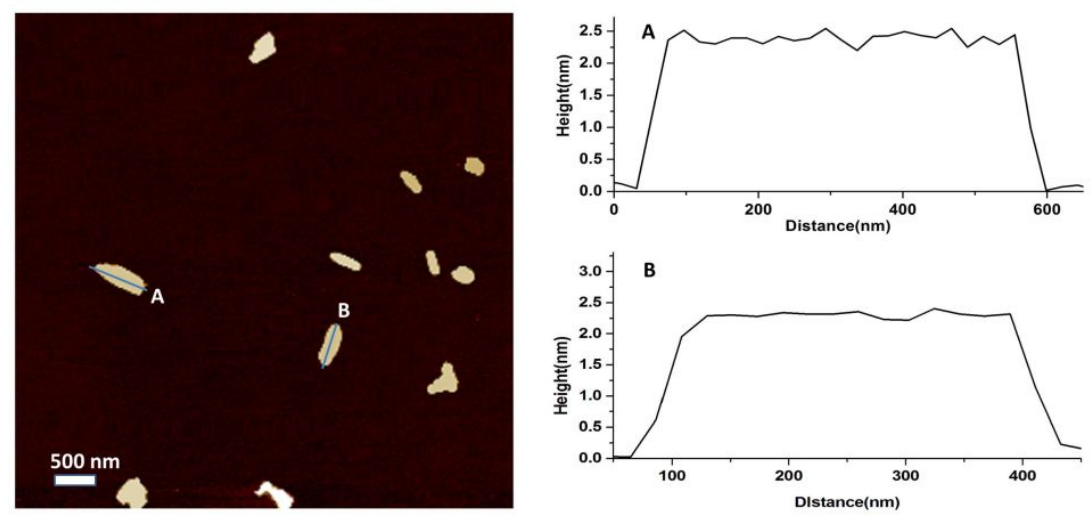

Figure S9. AFM images and corresponding thickness for the nanosheets of a, 1, and $\mathbf{b}, \mathbf{2}$, demonstrating the homogeneity of the nanosheets. 


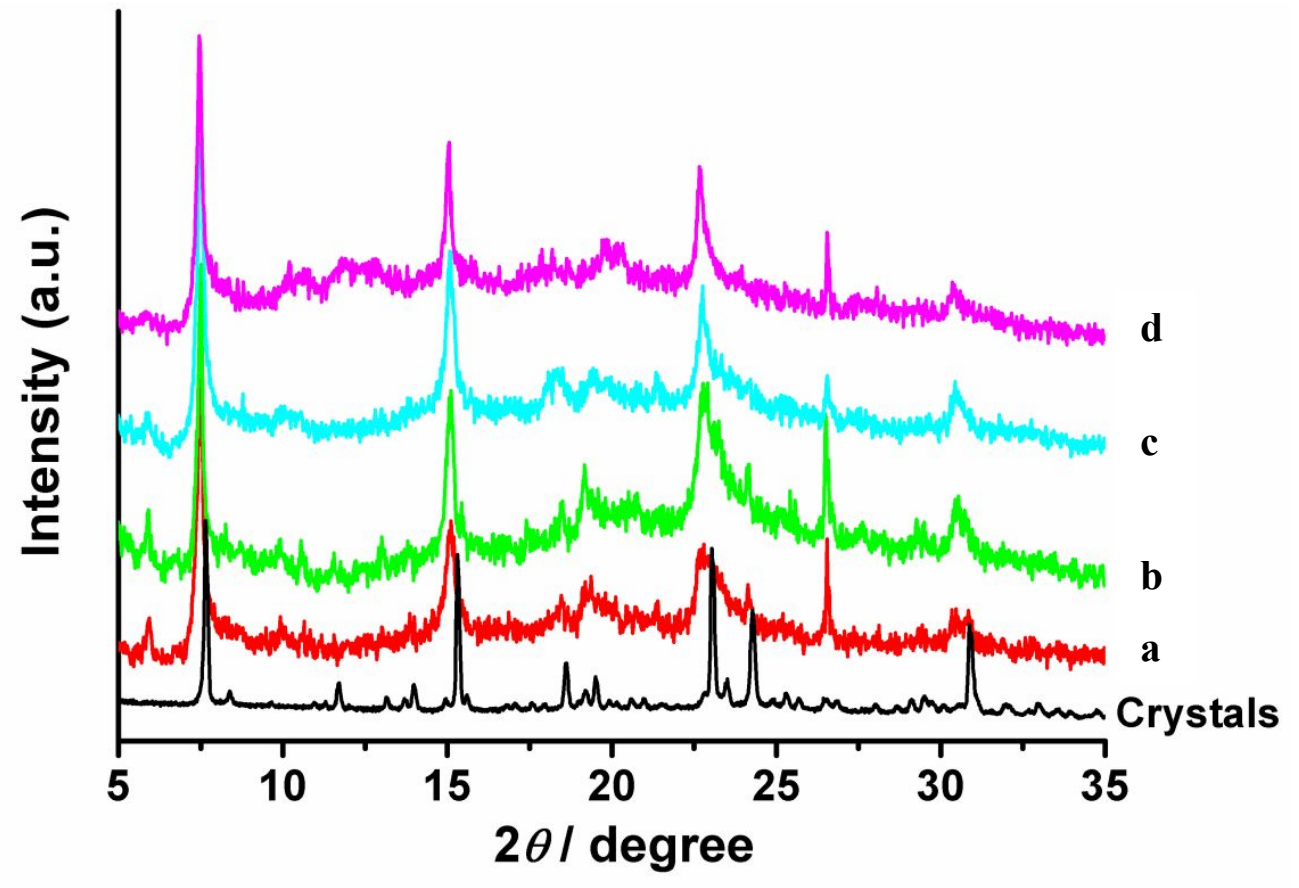

Figure S10. XRD patterns of nanosheets of 1 using different replacing agents a, 4-pyridylamine and 2,6-diaminopyridine, b, 4-pyridylamine and 3,5-diaminopyridine, c, 4-pyridylamine and 2,4,6-triaminopyridine, as well as d, 4-pyridylamine, 2,6-diaminopyridine and 2,4,6-triaminopyridine. 


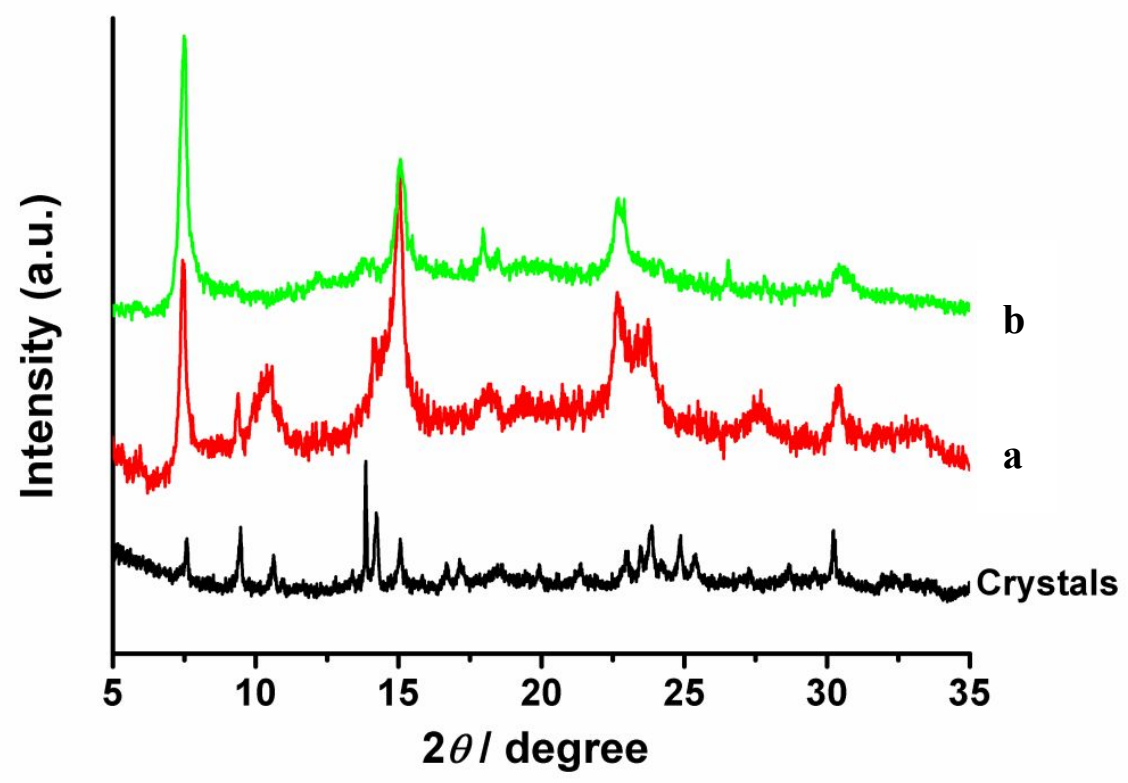

Figure S11. XRD patterns of nanosheets of 2 using different replacing agents a, 2,6-dimethylpyridine, and b, 3,5-dimethylpyridine. 

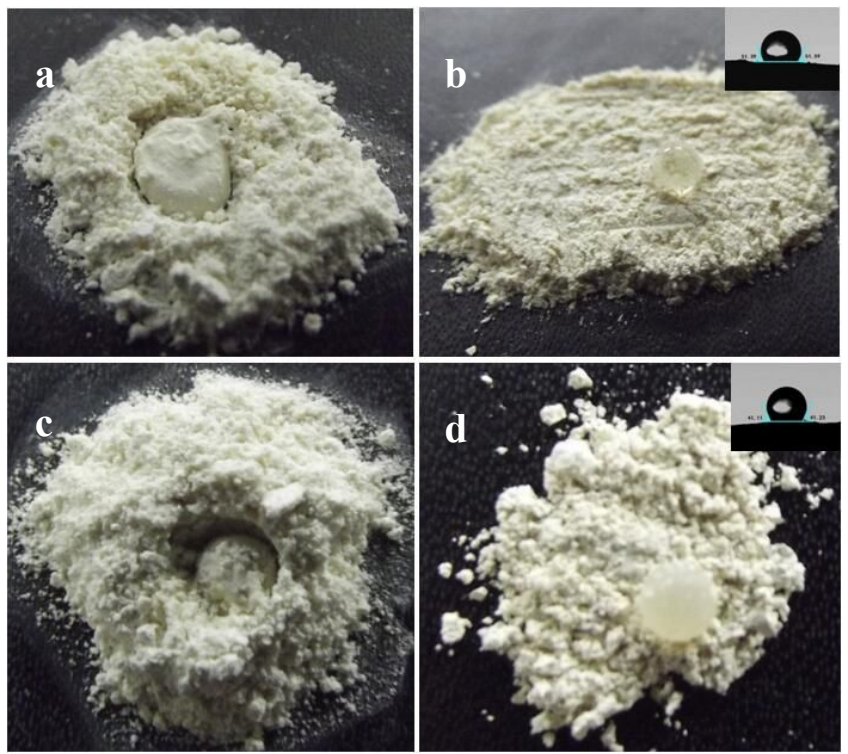

Figure S12. Digital photographs of a, 1, b, nanosheets of 1, c, 2, and d, nanosheets of $\mathbf{2}$ after a drop of water was placed onto the samples. Inset in $\mathbf{b}$ and $\mathbf{d}$ shows contact angle measurement for nanosheets of $\mathbf{1}$ and $\mathbf{2}$, respectively. 
a

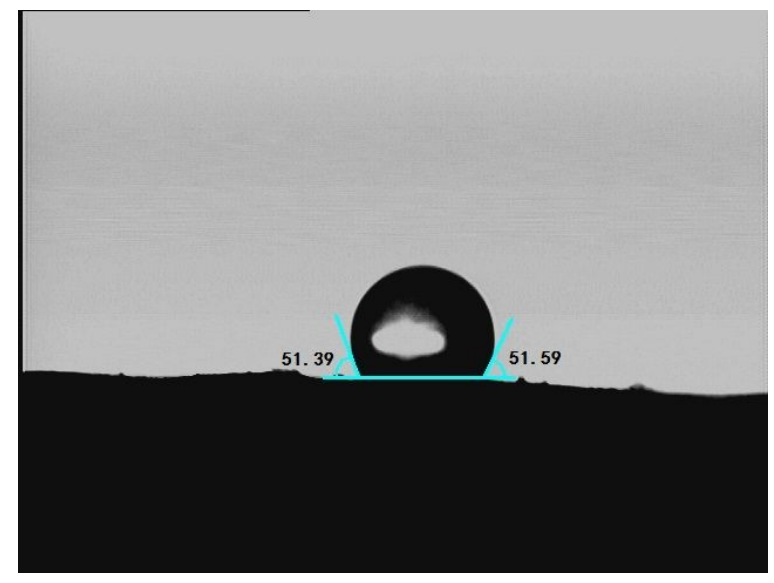

b

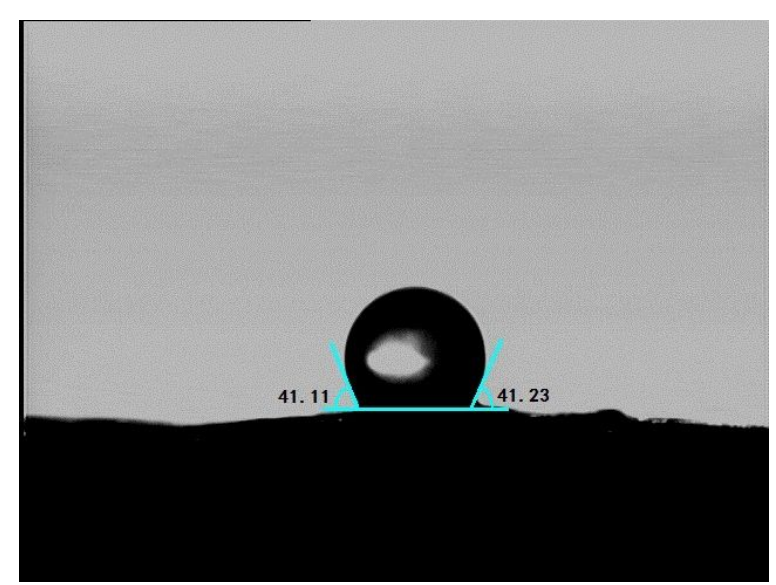

Figure S13. Contact angle measurements for nanosheets of $\mathbf{a}, \mathbf{1}$ and $\mathbf{b}, \mathbf{2}$. 

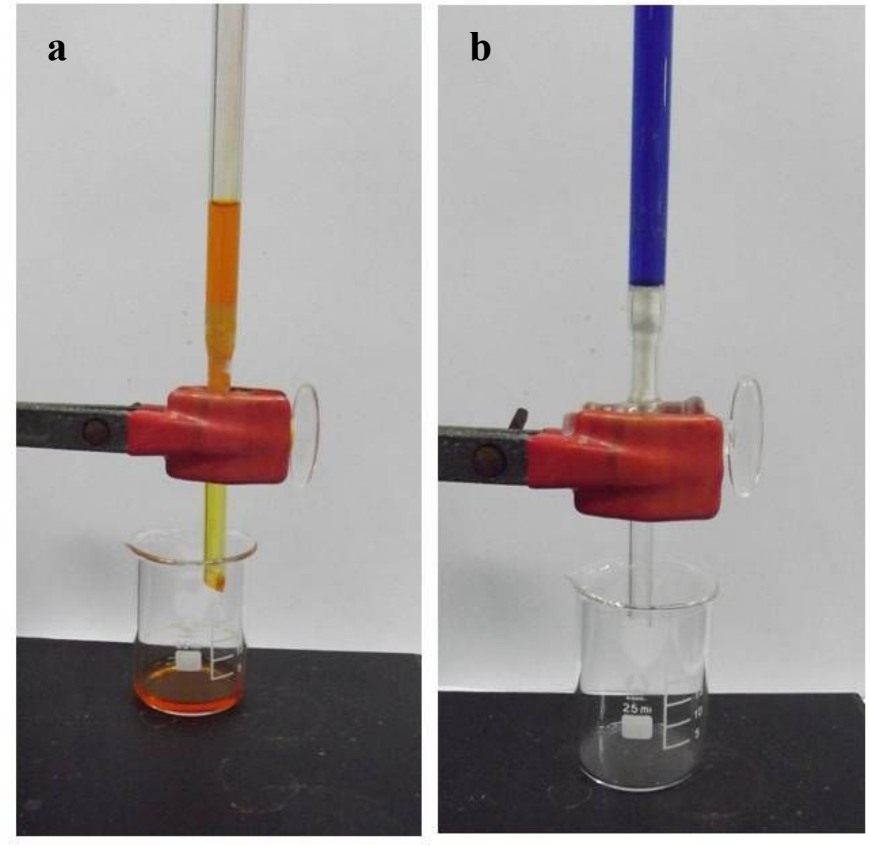

Figure S14. Digital photographs showing the filtrate tests of MOF nanosheets 1 for a, oil (cyclohexane, stained with Sudan red), and b, water (stained with methylene blue), where it can be seen that the oil in orange can well pass through the MOF nanosheet layer, while the water in blue can not pass through the MOF nanosheet layer. 